\title{
Neutrophils infiltration is early event of an oral mucosal xenotransplantation model
}

\author{
Hong Wang ${ }^{1}$, Xiao-An Tao ${ }^{1}$, Jin Xiang ${ }^{2}$, Juan Xia ${ }^{1}$, Yulei Huang ${ }^{1}$, Bin Cheng ${ }^{1}$ \\ ${ }^{1}$ Guanghua School of Stomatology, Sun Yat-sen University, Guangzhou, PR China, 510060 \\ ${ }^{2}$ Department of Pathology, Sun Yat-sen University Cancer Center, Guangzhou, PR China, 510061
}

Correspondence:

Guanghua School of Stomatology,

Sun Yat-sen University,

Guangzhou, PR China

chengbin@mail.sysu.edu.cn

Received: 08/06/2010 Accepted: 14/11/2010

\author{
Wang H, Tao XA, Xiang J, Xia J, Huang Y, Cheng B. Neutrophils infil- \\ tration is early event of an oral mucosal xenotransplantation model. Med \\ Oral Patol Oral Cir Bucal. 2011 May 1;16 (3):e341-7. \\ http://www.medicinaoral.com/medoralfree01/v16i3/medoralv16i3p341.pdf \\ Article Number: $17140 \quad$ http://www.medicinaoral.com/ \\ (C) Medicina Oral S. L. C.I.F. B 96689336 - pISSN 1698-4447 - eISSN: 1698-6946 \\ eMail: medicina@medicinaoral.com \\ Indexed in: \\ Science Citation Index Expanded \\ Journal Citation Reports \\ Index Medicus, MEDLINE, PubMed \\ Scopus, Embase and Emcare \\ Indice Médico Español
}

\begin{abstract}
Introduction: As important effector cells of the innate immune system, neutrophils are involved in rejection of solid organ and/or tissue transplants. But their role in rejection of oral mucosa transplantation (OMT) remains unclear. The aim of this study is to observe the spatial-temporal change of neutrophils during acute rejection of OMT.

Methods: In a rat model of oral mucosal xenotransplantation, myeloperoxidase (MPO), an indicator of influx of neutrophils was detected by technique of ELISA on day 7 and 30 (D7, D30) of posttransplantation.

Results: On D7, MPO level $\left(6.183 \pm 0.416, \times 10^{2} \mathrm{ng} / \mathrm{mg}\right)$ in the OMT group was significantly higher than in trauma $\left(0.681 \pm 0.073, \times 10^{2} \mathrm{ng} / \mathrm{mg}\right)$ and normal controls $\left(0.262 \pm 0.043, \times 10^{2} \mathrm{ng} / \mathrm{mg}\right)(P<0.001$, respectively), and this level was found to correlate with the index of submandibular lymph nodes (ILN), an indicator of inflammation of rejection $(r=0.909, P<0.05)$. Moreover, this level was decreased significantly under FK506 treatment $(2.103 \pm 0.146$, $\left.\times 10^{2} \mathrm{ng} / \mathrm{mg}, P=0.005\right)$. On D30, MPO in the OMT group $\left(1.063 \pm 0.096, \times 10^{2} \mathrm{ng} / \mathrm{mg}\right)$ was lower significantly than that on D7 $(P<0.001)$, although this level was still higher that of normal controls on D30 $\left(0.532 \pm 0.112, \times 10^{2} \mathrm{ng} /\right.$ $\mathrm{mg}, P=0.042)$.

Conclusion: Neutrophils infiltration was an early event of OMT, which may play important roles on acute rejection of OMT.
\end{abstract}

Key words: Oral mucosa xenotransplantation, neutrophils, myeloperoxidase, tacrolimus.

\section{Introduction}

Neutrophils are important effectors cells of the innate immune system (IIS) and could also play as modulators between IIS and the adaptive immune system (AIS). Numerous studies have shown that neutrophils play important roles in the regulation of immunological processes involving in transplant rejection via several mechanisms, including production of reactive oxygen species (ROS) and pro-inflammatory cytokines and chemok- ines (1-3). Neutrophils were the main infiltrating cells in the early phases of skin or corneal graft rejections $(4,5)$. The increased neutrophils and their chemokines were involved in liver inflammation after hepatocyte transplant (6). Clinically, the effect of ulinastatin, a protease inhibitor, on ischemia-reperfusion injury was correlated with decreasing accumulation and adhesion of neutrophils in small bowel transplant (7). The number of neutrophils in broncho-alveolar lavage fluid was an 
indicator of aggravated lung rejection or infection (8). Importantly, The efficiency of immunosuppression to reduce rejection rates and prolong graft survival times could be attributed, in part, to decreased phagocytic capacity and the generation of ROS by neutrophils (9). All the evidences indicated that understanding the role of neutrophils in transplant rejection will provide some new insights into the mechanisms of transplantation immunity and its clinical potentials.

However, in the field of oral-facial transplant, available data on the role of neutrophils have little been reported by now. Being scarce of suitable animal models may be the main block. As well known, in the oral-facial field, establishing a model of composite tissue transplantation (CMT) is so complex and difficult. Compared with CMT, oral mucosal transplant (OMT) is simple and easy to be manipulated. In addition, researches on OMT would provide some clues or references for CMT in clinic. So suitable OMT model was necessary and may be a useful tool for investigating transplant immunity in oral-facial field. However, available models of OMT either use nude mice as recipients (10-12) or belong to a kind of syngenesioplastic gum transplantation (13-15). Genetic background of nude mice could not reflect the natural immunological features of animals with normal immune ability and syngenesioplastic status was not with immune involved. OMT essentially belongs a kind of epithelial tissue transplantation (16), but as for complex micro-organism influences, kinds of immune defense mechanisms of oral cavity $(17,18)$ and strikingly regionalized effecter disparity in human mucosal immune system (19), researches on other epithelial tissue transplant such as skin or cornea could just be as reference but could not be applied directly to oral mucosa. Therefore, immunological response of OMT needs to be explored particularly.

Recently, an oral mucosal xenotransplant model with normal rats as recipients had been established primarily in our laboratory and on the foundation of this model, it was found that neutrophils infiltration was predominant, especially at the early stage (unpublished data). So the aim of this pilot study was to explore the possible role of neutrophils infiltration at the early phase of OMT.

\section{Materials and Methods \\ -Experimental design}

Animal models of OMT, trauma control and normal control were established. Some rats were sacrificed on D7 or D30 and symptoms of rejection were observed grossly and histopathology. Meloperoxidase (MPO) levels on D7 and D30 were detected by technique of ELISA analysis. Altersations of rejection symptoms and MPO level on D7 were also noted after FK506 treatment. Animals

Female Wistar rats weighing $160 \mathrm{~g}-180 \mathrm{~g}$ as recipients and female Balb/C mice weighing $18 \mathrm{~g}-20 \mathrm{~g}$ as donors were purchased from the center of Experimental Animals of Sun Yat-sen University (Guangzhou, China) and bred in a specific pathogen-free environment with climate-controlled rooms. All experimental protocols were approved by the Animal Ethics Committee and were performed according to institutional guidelines of the University.

\section{-Xenotransplantation and FK506 treatment}

Briefly, an incision no more than $1 \mathrm{~cm}$ long was made on the left angle oris of Wistar rats, and blunt dissection inside the incision was performed under the buccal mucosa to form the recipient bed, in which the full tongue tissue with $0.3-0.5 \mathrm{~cm}$ length from Balb/C mice was embedded. Then, the incisions were sutured carefully. Rats that received similar surgical procedures but without embedding of tongue tissues and rats that not received surgical procedures were assigned as trauma and normal controls, respectively.

Some rats of xenoragft $(\mathrm{X})$, trauma $(\mathrm{T})$ and normal $(\mathrm{N})$ controls groups, were pre-treated with high doses $(2 \mathrm{mg}$ ) $\mathrm{kg} /$ day, orally) of FK506 (Fujisawa Pharmaceutical Co, $0.5 \mathrm{mg} / \mathrm{pill}$ ) for 3 consecutive days before surgery and then after surgery they received low doses $(0.5 \mathrm{mg} / \mathrm{kg} /$ day, orally) of FK506 for 7 consecutive days until they were sacrificed on D7. FX, FT and FN indicate above 3 groups with treatment of FK506 respectively. Animal groups and treatments are summarized in table 1.

-Gross and histopathological observations

Swelling and pyorrhea of the left buccal mucosa were observed. Submandibular lymph nodes (SMLN) on both sides were isolated and an index of SMLN (ILN) was calculated: ILN $=$ sum of diameters of the 3 largest left SMLN ${ }^{-}$sum of diameters of the 3 largest right SMLN(taking the absolute value). ILN directly reflected the degree of the left SMLN swelling and indirectly reflected the degree of rejection.

Whole buccal tissues of rats in each group were isolated. One-half samples were fixed with $10 \%$ formaldehyde, embedded in paraffin by standard protocols, serially sectioned (4 $\mu \mathrm{m})$, stained with hematoxylin-eosin (HE) and made histological analysis by 2 experienced

Table 1. Experimental Rat Groups and Treatments.

\begin{tabular}{|l|l|l|l|}
\hline \multicolumn{1}{|c|}{ Groups } & \multicolumn{1}{c|}{ D7 } & D30 & Total \\
\hline Xenograft group (X) & 12 & 12 & 24 \\
\hline Trauma group (T) & 12 & 12 & 24 \\
\hline Normal Group ( N) & 8 & 8 & 8 \\
\hline Fk506+X group (FX) & 12 & ND & 12 \\
\hline Fk506+T group (FT) & 12 & ND & 12 \\
\hline Fk506+N group (FN) & 8 & ND & 8 \\
\hline
\end{tabular}

Entries are numbers in each category; ND: not done. 
pathologists. The other half samples were stored in liquid nitrogen until MPO detection.

\section{-MPO detection}

(1) Extraction of tissue protein: The buccal mucosal tissues (about $300 \mathrm{mg}$ ) were triturated with liquid nitrogen and were homogenized in $6 \mathrm{ml}$ T-PER tissue protein extraction reagent (Pierce, Rockford, IL, USA). Sample homogenates were centrifuged at $10,000 \mathrm{~g}$ for 5 minutes at $4^{\circ} \mathrm{C}$. Supernatants were stored at $-80^{\circ} \mathrm{C}$ until used.

(2) Measurement of total protein: Total protein in homogenate liquids was quantified using a BCA (Bicinchoninic Acid) Protein Assay Kit (Pierce, Rockford, IL, USA) according to the manufacturer's protocol. Sample absorbance was measured at $562 \mathrm{~nm}$ within 10 minutes,

\section{Results}

-Gross and histological observation

(1) Groups on D7

X-D7 group: Swelling and pyorrhea of the left buccal mucosa were obvious, and erosion sometimes could be seen; the left SMLN (xenograft side) was swollen obviously (Fig.1.A). After the treatment of FK506, only mild swelling with little pyorrhea of the left buccal mucosa and mild to moderate swelling of the left SMLN were manifested (Fig1.C). ILN increased significantly compared with T-D7, N-D7 and X-D30 groups (Table 2) and protein concentration was determined from a standard curve.

(3) ELISA analysis of MPO: The concentration of MPO in supernatants was determined using an ELISA kit (HyCult Bio- technology, Uden, Netherlands) as previously described (20). The concentration of MPO in tissue was expressed as the ratio of MPO concentration to total protein in order to take into account slight differences of tissue weights (21). So the level of MPO was presented as $\mathrm{ng} / \mathrm{mg}$ in tissues.

-Statistics

Data collection and statistical analysis were performed using SPSS 13.0 statistical software (SPSS Inc, Chicago, IL, USA). The results are given as mean \pm standard deviation (SD). The analysis of variance (ANOVA) was used to compare quantitative measurements. Association of MPO level and ILN were assessed by spearman correlation coefficient. $P<0.05$ was considered as statistically significant for all statistical test carried out. After the treatment of FK506, ILN decreased significantly (Table 2). HE staining showed that in the xenograft site, part or whole coagulation necrosis with intense neutrophils infiltration appeared and abscess formed; outsides the abscess, fibroblasts and capillaries hyperplasia were accompanied by large number of lymphocytes, plasma cells, histiocytes, and occasionally a few polynuclear-macrophages(Fig.1.A). After the treatment of FK506, histological structure of the xenograft sites was almost intact, mild necrosis was accompanied just by mild to moderate infiltrations of neutrophils (Fig.1.C).

T-D7 group: Minor or no swelling and pyorrhea of the left buccal mucosa were manifested; the left SMLN (trauma side) was swollen slightly or moderately (Fig.1.D). ILN increased significantly compared with N-D7 and T-D30 groups (Table 2). HE staining showed that there remained minor fissures in buccal striated muscle and minor interstitial edema was accompanied by a slight infiltration of plasmacytes and histiocytes (Fig.1.D). After the treatment of FK506, rejection symptoms and HE staining were similar with T-D7 group, but ILN decreased significantly (Table 2).

N-D7 group: Normal appearance and histological structures of the left buccal mucosa were manifested; the left SMLN (normal side) was not swollen (Fig.1.E); ILN showed no significant difference with that in N-D30 group (Table 2). After the treatment of FK506, no obvious alterations of above symptoms could be found.

(2) Groups on D30

Swelling and pyorrhea of the left buccal mucosa and swelling of the left SMLN in each group on D30 were no longer obvious (Fig1.B); ILN showed no significant differences between any other two groups on D30 (Table 2). HE staining showed that in X-D30 group, tissue repair began to be predominant and numerous fibroplasias surrounded the necrosis sites with moderate infiltrations of histiocytes, polynuclear-macrophages, plasmocytes, small numbers of lymphocytes and a few neutrophils (Fig.1.B); in T-D30 group, the trauma site had almost healed, sometimes accompanied with little plasma cells, histiocytes; in N-D30 group, normal appearance and normal histological structures were manifested.

-MPO levels

(1) Groups on D7

MPO level in X-D7 group increased significantly compared with the T-D7, N-D7 and X-D30 groups; it in T-D7 group was higher than N-D7 group; there was no significant difference between N-D7 and N-D30 groups or T-D7 and T-D30 groups. After the treatment of FK506, MPO level in FX-D7 group decreased significantly compared with X-D7group, but it was still higher than that in FT-D7 and FN-D7 groups (Table 3).

(2) Groups on D30

MPO level in X-D30 group decreased significantly compared with X-D7 group, but was still higher than that in N-D30 group (Table 3).

Correlation analysis between MPO levels and ILN Only in X-D7 group, MPO level and ILN performed positive correlation; after the treatment of FK506, this positive correlation was not significant any more (Table 4). 


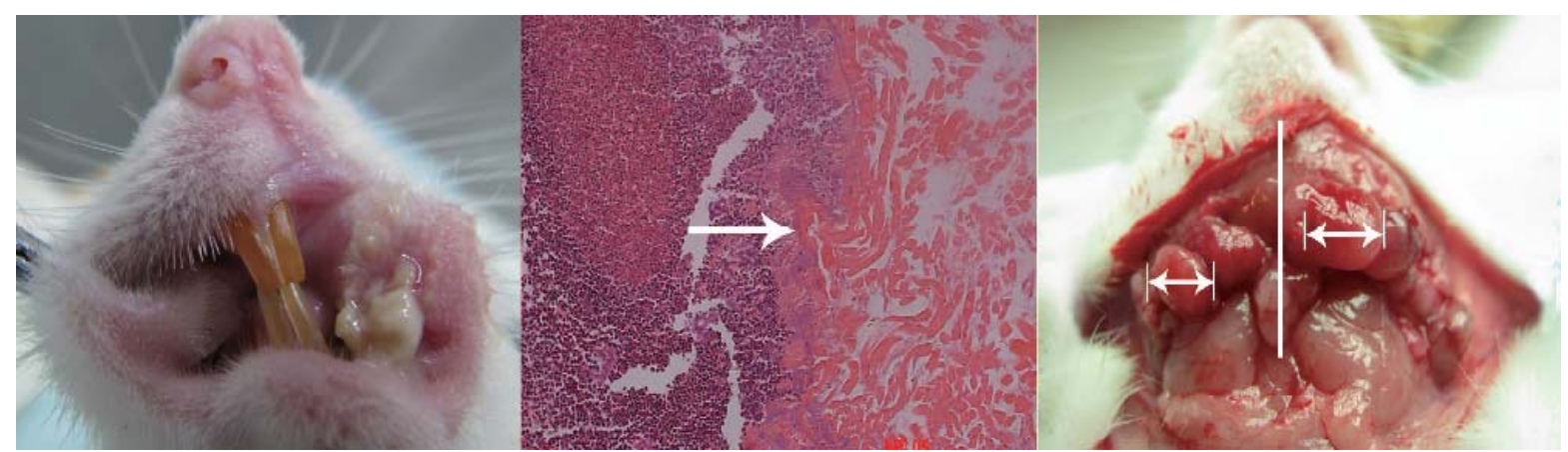

Fig. 1. A) X-D7 group.

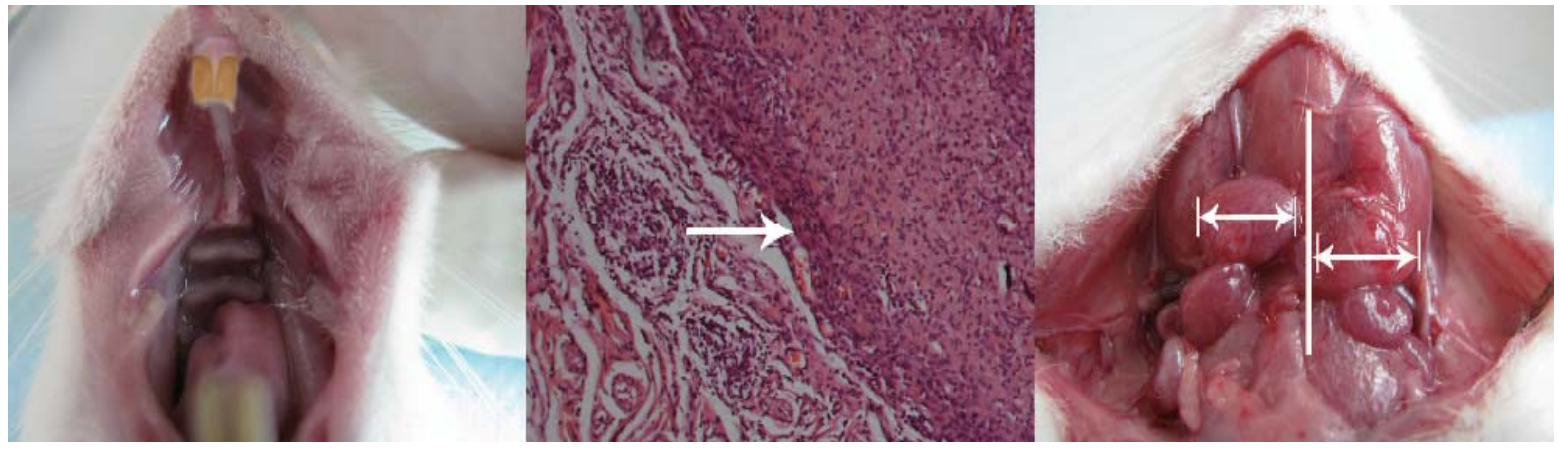

Fig. 1. B) X-D30 group.
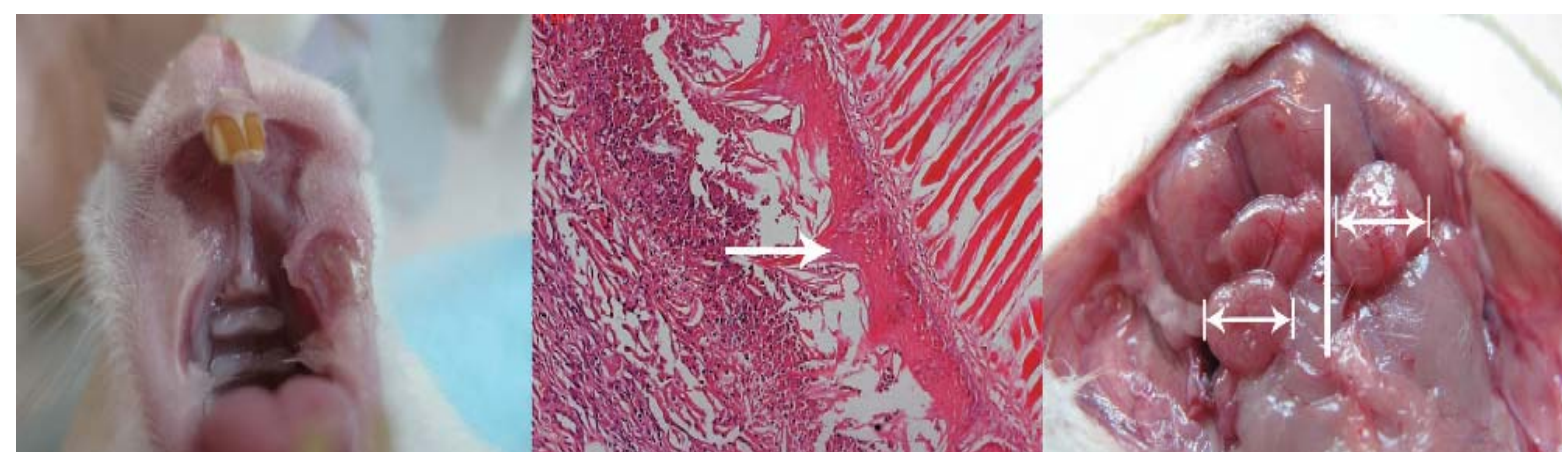

Fig. 1. C) FX-D7 group.
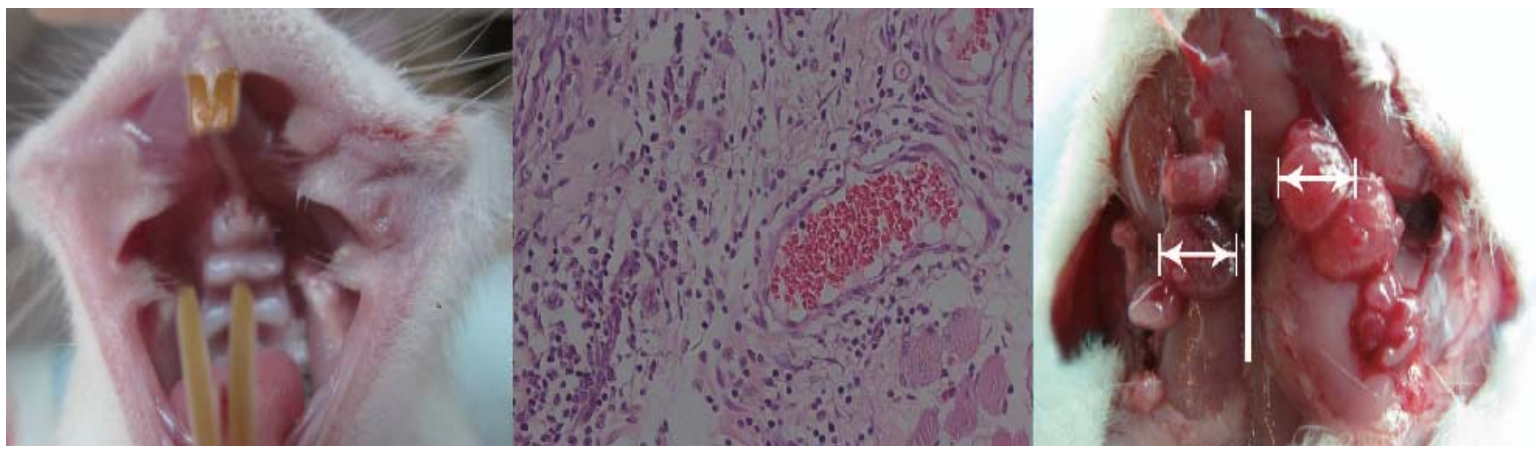

Fig. 1. D) T-D7 group. 

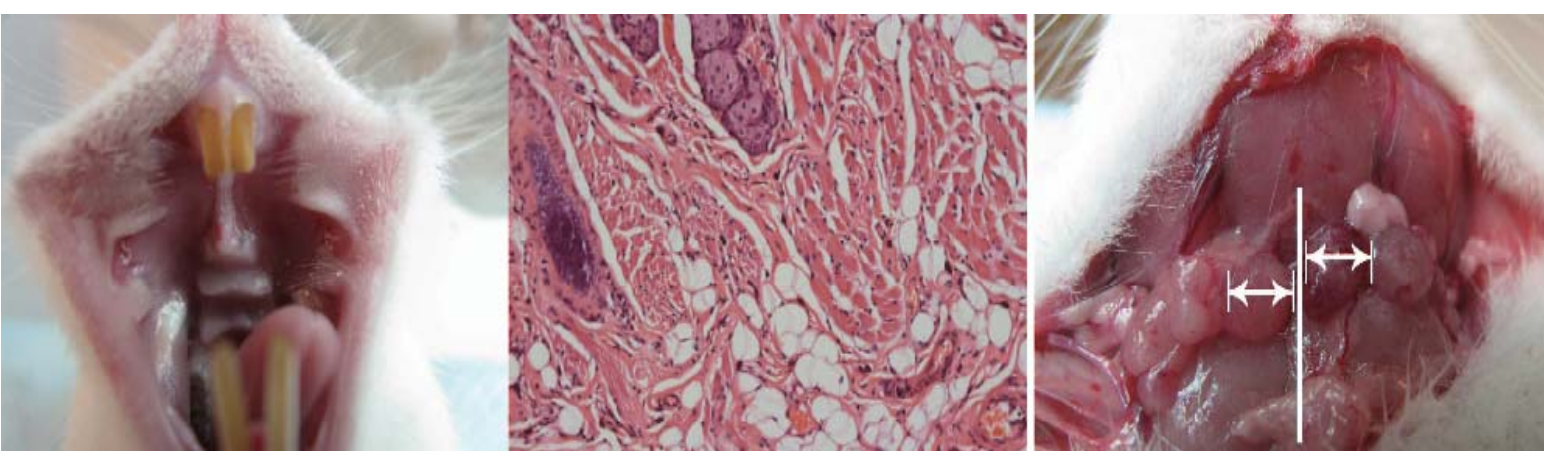

Fig. 1. E) N-D7 group.

Fig. 1. Gross and histopathological observations on the buccal mucosa and SMLN (submandibular lymph nodes) of the different groups on D7 and D30. The left column: swelling and pyorrhea of the buccal mucosa; the right column: swelling of SMLN (arrow indicates the diameter of a SMLN in each side); the middle column: HE staining of the left buccal mucosa (arrow indicates boundary of the xenograft) (X200).

Table 2. ILN (mm) of Groups on D7 and D30 with or without FK506 Treatment.

\begin{tabular}{|c|c|c|c|c|c|}
\hline \multirow[b]{2}{*}{ Groups } & \multicolumn{2}{|r|}{ D7 } & \multirow{2}{*}{$\begin{array}{c}\text { D30 } \\
\text { WithoutFK506 } \\
\mathbf{\Delta} \boldsymbol{\Delta} \boldsymbol{\Delta} \\
\end{array}$} & \multicolumn{2}{|c|}{$P$ values } \\
\hline & $\begin{array}{c}\text { With FK506 } \\
\Delta\end{array}$ & 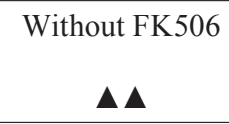 & & $\boldsymbol{\Delta} \sim \boldsymbol{\Delta} \boldsymbol{\Delta}$ & $\boldsymbol{\Delta} \boldsymbol{\Delta} \sim \boldsymbol{\Delta} \boldsymbol{\Delta} \boldsymbol{\Delta}$ \\
\hline$X(n=6) *$ & $3.225 \pm 0.457$ & $5.841 \pm 0.106$ & $1.493 \pm 0.091$ & $P<0.001$ & $P<0.001$ \\
\hline $\mathrm{T}(\mathrm{n}=6)^{* *}$ & $2.240 \pm 0.162$ & $4.232 \pm 0.109$ & $1.678 \pm 0.104$ & $P=0.046$ & $P=0.046$ \\
\hline $\mathrm{N}(\mathrm{n}=4) * * *$ & $1.650 \pm 0.192$ & $2.160 \pm 0.081$ & $1.578 \pm 0.479$ & $P=0.095$ & $P=0.795$ \\
\hline$* \sim * *$ & $P=0.048$ & $P=0.038$ & $P=0.118$ & & \\
\hline$* \sim * * *$ & $P=0.001$ & $P<0.001$ & $P=0.766$ & & \\
\hline$* * \sim * * *$ & $P=0.047$ & $P<0.001$ & $P=0.691$ & & \\
\hline
\end{tabular}

Values are mean \pm standard deviation (SD). ILN = sum of diameters of the 3 largest left SMLN - sum of diameters of the 3 largest right SMLN (taking the absolute value).

(X; xenograft group, T: trauma group, N: normal group).

Table 3. MPO Levels of Groups on D7 and D30 with or without FK506 Treatment (Unit: $\times 10^{2}$ ng MPO/ mg total proteins).

\begin{tabular}{|c|c|c|c|c|c|}
\hline \multirow[b]{2}{*}{ Groups } & \multicolumn{2}{|r|}{ D7 } & \multirow{2}{*}{$\begin{array}{c}\text { D30 } \\
\text { Without FK506 } \\
\mathbf{\Delta} \mathbf{\Delta} \mathbf{\Delta}\end{array}$} & \multicolumn{2}{|c|}{$P$ values } \\
\hline & $\begin{array}{c}\text { With FK506 } \\
\text { 口 }\end{array}$ & Without FK506 & & $\boldsymbol{\Delta} \sim \boldsymbol{\Delta} \mathbf{\Delta}$ & $\Delta \mathbf{\Delta} \sim \Delta \mathbf{\Delta} \Delta$ \\
\hline$X(n=6) *$ & $2.103 \pm 0.146$ & $6.183 \pm 0.416$ & $1.063 \pm 0.096$ & $P=0.005$ & $P<0.001$ \\
\hline $\mathrm{T}(\mathrm{n}=6)^{* *}$ & $1.032 \pm 0.416$ & $0.681 \pm 0.073$ & $0.532 \pm 0.112$ & $P=0.184$ & $P=0.086$ \\
\hline$N(n=4) * * *$ & $0.323 \pm 0.069$ & $0.262 \pm 0.043$ & $0.403 \pm 0.110$ & $P=0.997$ & $P=0.995$ \\
\hline$* \sim * *$ & $P=0.029$ & $P<0.001$ & $P=0.526$ & & \\
\hline$* \sim * * *$ & $P=0.001$ & $P<0.001$ & $P=0.042$ & & \\
\hline$* * \sim * * *$ & $P=0.015$ & $P=0.019$ & $P=0.998$ & & \\
\hline
\end{tabular}

Values are mean \pm standard deviation (SD). MPO level in tissue $=$ MPO concentration $/$ total protein concentration. (X; xenograft group, T: trauma group, N: normal group). 
Table 4. Correlation between MPO Levels and ILN of Groups on D7 and D30.

\begin{tabular}{|c|c|c|}
\hline \multirow{2}{*}{ Groups } & \multicolumn{2}{|c|}{ MPO levels and ILN } \\
\cline { 2 - 3 } & $\begin{array}{c}\text { Correlation } \\
\text { coefficient }(\boldsymbol{R})\end{array}$ & P value \\
\hline X-D7 $(\mathrm{n}=6)$ & 0.909 & $0.012^{*}$ \\
\hline T-D7 $(\mathrm{n}=6)$ & 0.736 & 0.095 \\
\hline N-D7 $(\mathrm{n}=4)$ & 0.574 & 0.426 \\
\hline X-D30 $(\mathrm{n}=6)$ & 0.762 & 0.078 \\
\hline T-D30 $(\mathrm{n}=6)$ & 0.415 & 0.414 \\
\hline N-D30 $(\mathrm{n}=4)$ & 0.413 & 0.862 \\
\hline FX-D7 $(\mathrm{n}=6)$ & 0.806 & 0.053 \\
\hline FT-D7 $(\mathrm{n}=6)$ & 0.718 & 0.108 \\
\hline FN-D7 $(\mathrm{n}=4)$ & 0.814 & 0.161 \\
\hline
\end{tabular}

* means the correlation coefficient was statistical significance. (X; xenograft group, T: trauma group, N: normal group).

\section{Discussion}

Several lines of evidences have proven the roles of neutrophils in graft rejection of some organs and/or tissues transplants. As effector cells, neutrophils, either alone or in collaboration with monocytes, directly destruct grafts by production of superoxide anions with high activities and granzymes, including protease, elastase and lysozyme $(22,23)$. More importantly, as connectors, neutrophils secrete a lot of inflammatory cytokines or chemokines, including TNF- $\alpha$, IL-8, MIP- $1 \alpha / \beta$ (CCL3, CCL4) and IP-10 (CXCL10) etc in an auto- or paracrine manner to initiate the recruitment of immune cells of IIS and AIS to the graft sites, which lead to the last rejection of graft $(24,25)$. Therefore, increasing attention has been paid to the roles of neutrophils during rejection. However, the role of neutrophils in oral-facial transplant has not been reported by far. One of the main bottlenecks was partly due to being scarce of suitable animal models.

Recently, a developed oral mucosal xenotransplant model, which with normal rats as recipients, had been established primarily in our laboratory. Xenotransplant model was chosen as it was demonstrated at the preliminary experiment that the symptoms of acute rejection of OMT between xenotransplant and allotransplant was similar but earlier and more intensive rejection symptoms would be induced by the former. For a successive 90-days gross and histopathological observation, neutrophils infiltration was found to be predominant at the early phase and to disappear obviously at the late phase; especially on D7 and D30 (unpublished data). So D7 and D30 may represent the acrophase and paracmasia of inflammation in this OMT model respectively. Accordingly, the degrees and roles of neutrophils infiltration of OMT on D7 and D30 were noted in this pilot study. At present study, MPO levels which reflect the degree of neutrophils influx into tissues (26), of buccal mucosa on D7 and D30 were compared for confirming the different influx of neutrophils. It was found that MPO level of X-D7 group was significantly higher than that of on D30 and this higher MPO level could be attenuated by the treatment of immunosuppressant FK506. The same circumstance could also be found on ILN of X-D7 group, which indirectly reflects the degree of rejection of OMT and other acute rejection symptoms. FK506 have been proved to inhibit transplant rejection and prolong the survival of allografts by inhibiting the infiltration of neutrophils $(27,28)$. So the inhibition role of FK506 further confirmed the role of neutrophils in promoting rejection of OMT. It seems the first time, for the best of our knowledge, to demonstrate the participation of neutrophil infiltration in rejection of oral mucosal xenotransplant. In addition, MPO level and ILN of the xenograft group on D7 performed a positive correlationship, which indicates that neutrophils infiltrating has tight relationship with the rejections of OMT. The same opinion could also be supported by the gross and histopathological observations. From all of above evidences, it was concluded that in the early phase of OMT, neutrophils infiltration was predominant and as effector cells, neutrophils played key roles on promoting the rejection of OMT.

Neutrophils could also modulate rejections of other organs and tissues transplants. In organ transplants, neutrophils could infiltrate into lungs at 6 hours in a rat lung transplantation model (8); it was verified to increased at about 2 hours after revascularization in a rat small bowel transplantation model (7); an increase of neutrophil chemoattractant CINC-1/interleukin-8 (IL-8) mRNA expression was associated with a marked infiltration of neutrophils in renal tissue at 2 hours in a rat model of ischemia/reperfusion injury followed by kidney transplant (29) and neutrophil chemoattractant growth-related oncogene alpha $(\mathrm{KC})$ expression peaked at $6 \mathrm{~h}$ and neutrophils infiltration increased significantly 48 hours after transplant in a mice heterotopical allograft heart transplantation model (30). In other tissues transplants, neutrophils can also often be found in the early stages post-transplantation. Predominantly infiltrating of neutrophils were found on day 3 in a rat-tomouse skin xenograft model (4); on day 3 in a corneal allograft model (5), on day 4 in a porcine-to-rat corneal orthotopic transplantation model (28) and from day 1 onward in this OMT model (unpublished data). Therefore, like other organs or tissues transplants, neutrophils infiltration is an early event of OMT and it maybe play key roles on promoting the acute rejection of OMT or oral-facial transplantation.

Our main findings will give some clues to understanding the role of neutrophils and its clinical potentials in oral-facial transplantation. For example, another ex- 
periment designed to explore the mechanisms of neutrophils recruitment in OMT have been completed by our group (unpublished data), which would bring some new insights for targeting inhibition of the rejections of oral-facial transplant; the degree of neutrophils infiltration could be used as a mainstay for pathological grading and detection of the neutrophils in saliva would be a convenient method to monitor the degree of rejections of oral-facial transplant as detecting neutrophils in the broncho-alveolar lavage fluid to reflect the degrees of lung rejection (7).

In summary, neutrophils infiltration is an early event of OMT. Therefore, exploring the roles of neutrophils during OMT would provide some new insights into the mechanisms of transplant immunology and its clinical potentials of the oral-facial field.

\section{References}

References with links to Crossref - DOI

1. Kobayashi Y. The role of chemokines in neutrophil biology. Front Biosci. 2008;13:2400-7.

2. Belperio JA, Keane MP, Burdick MD, Gomperts BN, Xue YY, Hong $\mathrm{K}$, et al. CXCR2/CXCR2 ligand biology during lung transplant ischemia-reperfusion injury. J Immunol. 2005;175:6931-9.

3. Zarbock A, Polanowska-Grabowska RK, Ley K. Platelet-neutrophil-interactions: linking hemostasis and inflammation. Blood Rev. 2007;21:99-111.

4. Lee EM, Park JO, Kim D, Kim JY, Oh KH, Park CG, et al. Early up-regulation of $\mathrm{CXC}$-chemokine expression is associated with strong cellular immune responses to murine skin xenografts. Xenotransplantation. 2006;13:328-36.

5. Lee HI, Kim MK, Oh JY, Ko JH, Lee HJ, Wee WR, et al. The role of cyclosporine and mycophenolate in an orthotopic porcine-to-rat corneal xenotransplantation. J Korean Med Sci. 2008;23:492-501.

6. Krohn N, Kapoor S, Enami Y, Follenzi A, Bandi S, Joseph B, et al. Hepatocyte transplantation-induced liver inflammation is driven by cytokines-chemokines associated with neutrophils and Kupffer cells. Gastroenterology. 2009;136:1806-17.

7. Zhang XQ, Sun JL, Chen YJ, Ma R, Fan XH, Sun JZ. Amelioration of ischemia-reperfusion injury of transplanted small intestine by ulinastatin: effects on accumulation and adhesion of neutrophil. Transplant Proc. 2005;37:4464-6.

8. Vanaudenaerde BM, Wuyts WA, Geudens N, Nawrot TS, Vos R, Dupont LJ, et al. Broncho-alveolar lavage fluid recovery correlates with airway neutrophilia in lung transplant patients. Respir Med. 2008;102:339-47.

9. Hutchinson P, Chadban SJ, Atkins RC, Holdsworth SR. Laboratory assessment of immune function in renal transplant patients. Nephrol Dial Transplant. 2003;18:983-9.

10. Xie JF. [An experimental oral premalignant lesion transplanted to nude mice].

Zhonghua Kou Qiang Yi Xue Za Zhi. 1989;24:149-51, 191.

11. Holmstrup P, Dabelsteen E, Harder F. EDTA separation and recombination of epithelium and connective tissue of human oral mucosa. Studies of tissue transplants in nude mice. Exp Cell Biol. 1985;53:32-40.

12. Holmstrup P, Hansen IL, Harder F, Dabelsteen E. Identification of human connective tissue in transplant of human oral mucosa in nude mice. Acta Pathol Microbiol Immunol Scand A. 1984;92:31-7.

13. Tal H, Moses O, Zohar R, Meir H, Nemcovsky C. Root coverage of advanced gingival recession: a comparative study between acellular dermal matrix allograft and subepithelial connective tissue grafts. J Periodontol. 2002;73:1405-11.

14. Rahmani ME, Lades MA. Comparative clinical evaluation of acellular dermal matrix allograft and connective tissue graft for the treatment of gingival recession. J Contemp Dent Pract. 2006;7:63-70.
15. McGuire MK, Cochran DL. Evaluation of human recession defects treated with coronally advanced flaps and either enamel matrix derivative or connective tissue. Part 2: Histological evaluation. J Periodontol. 2003;74:1126-35.

16. Filipponi F, De Simone P, Saviozzi A, Bozzi G. Tissue procurement and transplantation: a Tuscany perspective. Transplant Proc. 2008;40:1811-3.

17. Holsen DS, Johannessen AC. [Diseases that affect both skin and oral mucosa]. Tidsskr Nor Laegeforen. 2006;126:1214-7.

18. Bay B, Hilliges M, Weidner C, Sandborgh-Englund G. Response of human oral mucosa and skin to histamine provocation: laser Doppler perfusion imaging discloses differences in the nociceptive nervous system. Acta Odontol Scand. 2009;67:99-105.

19. Brandtzaeg P, Farstad IN, Haraldsen G. Regional specialization in the mucosal immune system: primed cells do not always home along the same track. Immunol Today. 1999;20:267-77.

20. Roelofs JJ, Rouschop KM, Leemans JC, Claessen N, de Boer AM, Frederiks WM, et al. Tissue-type plasminogen activator modulates inflammatory responses and renal function in ischemia reperfusion injury. J Am Soc Nephrol. 2006;17:131-40.

21. Tao X, Huang Y, Li R, Qing R, Ma L, Rhodus NL, et al. Assessment of local angiogenesis and vascular endothelial growth factor in the patients with atrophic-erosive and reticular oral lichen planus. Oral Surg Oral Med Oral Pathol Oral Radiol Endod. 2007;103:661-9.

22. Meyer-Hoffert U. Neutrophil-derived serine proteases modulate innate immune responses. Front Biosci. 2009;14:3409-18.

23. Gangappa S, Kokko KE, Carlson LM, Gourley T, Newell KA, Pearson TC, et al. Immune responsiveness and protective immunity after transplantation. Transpl Int. 2008;21:293-303.

24. Scapini P, Lapinet-Vera JA, Gasperini S, Calzetti F, Bazzoni F, Cassatella MA. The neutrophil as a cellular source of chemokines. Immunol Rev. 2000;177:195-203.

25. LaRosa DF, Rahman AH, Turka LA. The innate immune system in allograft rejection and tolerance. J Immunol. 2007;178:7503-9.

26. Qualls JE, Tuna H, Kaplan AM, Cohen DA. Suppression of experimental colitis in mice by CD11c+ dendritic cells. Inflamm Bowel Dis. 2009; 15:236-47.

27. Oltean M, Pullerits R, Zhu C, Blomgren K, Hallberg EC, Olausson M. Donor pretreatment with FK506 reduces reperfusion injury and accelerates intestinal graft recovery in rats. Surgery. 2007;141:66777.

28. Bysterská P, Svozílková P, Farghali H. Effect of certain immunosuppressants on non-specific immunity cells in murine corneal grafts: study on early phases after transplantation. Physiol Res. 2007;56:603-10.

29. Cugini D, Azzollini N, Gagliardini E, Cassis P, Bertini R, Colotta $\mathrm{F}$, et al. Inhibition of the chemokine receptor CXCR2 prevents kidney graft function deterioration due to ischemia/reperfusion. Kidney Int. 2005;67:1753-61.

30. Morita K, Miura M, Paolone DR, Engeman TM, Kapoor A, Remick DG, et al. Fairchild RL. Early chemokine cascades in murine cardiac grafts regulate $\mathrm{T}$ cell recruitment and progression of acute allograft rejection. J Immunol. 2001;167:2979-84.

\section{Acknowledgments}

This study was supported by three grant from the National Natural Science Foundation of China (No. 30872874, No. 30672317), Guangdong Natural Science Fund (No. 8151008901000080) and the Priming Scientific Research Foundation for the Junior Teachers of Medicine in Sun Yat-sen Univercity (2008016). We greatly appreciated the assistance of Prof. Xiaohua Chen, Ruyao Li and Mrs. Ronghui Qin in the Department of Oral Pathology, Guanghua School of Stomatology, Sun Yat-sen University. 